Tribute

\title{
Reconocimiento a los editores RUC
}

\section{Primera editorial RUC Julio 1986}

\author{
Armando López López ${ }^{1,2}$ \\ 1 Sociedad Colombiana de Urología, Bogotá, Colombia \\ 2Editor RUC, 1986-1989, Sociedad Colombiana de Urología, Bogotá, \\ Colombia
}

Urol Colomb 2021;30(4):e227-e228.

Con la entrega del primer número de la revista de nuestra Sociedad Colombiana de Urología (SCU), se cumple un anhelo de sus miembros y una necesidad científica de nuestro país, llenando un vacío en la bibliografía médica nacional.

Ya era tiempo de tener un medio de comunicación propio para el intercambio de ideas entre sus miembros y, por qué no, a nivel internacional en el futuro. Son muchos los trabajos de gran contenido y calidad que son presentados en nuestros congresos y pocos se publican o son enviados a otros medios informativos nacionales o extranjeros y la mayoría duerme en el escritorio de su autor perdiéndose conceptos importantes, junto con el desconocimiento general de la calidad científica de nuestros urólogos.

Varios han sido los esfuerzos para realizar una publicación dentro de nuestra institución y no puedo seguir adelante sin mencionar los nombres de los doctores Gustavo Escobar R y Mario Giraldo Henao, que presentaron un "Boletín de Actividades" de la SCU por los años de 1967 y 1968; posteriormente, los doctores Jorge Cavelier G y Carlos de Vivero A se empeñaron en la tarea de publicar los resúmenes de los congresos (1970-1972-1974). Otros colegas, entre ellos el doctor Alonso Acuña C., promovieron la idea de crear una revista y posiblemente muchos otros esfuerzos se han intentado, pero estoy seguro que esto no es solo idea de unos entusiastas colegas sino la meta de todos los urólogos colombianos. Debe destacarse aquí también la decidida colaboración de los miembros de la directiva nacional y la incondicional ayuda intelectual y física del secretario ejecutivo, doctor Héctor Hugo Álvarez L para llevar a buen término la publicación de la revista.

Address for correspondence Armando López López, Sociedad Colombiana de Urología, Bogotá, Colombia (e-mail: revistaurologiacolombiana @scu.org.co).
Esperamos que al lograr presentar a ustedes esta publicación sea un motivo de estímulo para llenar sus páginas, tanto con los trabajos de los congresos como con la producción permanente de los colegas. Lo importante es que hemos iniciado y tenemos un largo y duro camino por recorrer, pero no podemos desfallecer, necesitamos el respaldo de todos los miembros de la familia urológica colombiana, muy especialmente con la producción científica propia; no aspiramos a mantener nuestra revista con base en trabajos extranjeros, estos trabajos tendrán cabida en ella, pero no debe ser la única fuente, ni su mayoría, lo que con frecuencia se observa en las publicaciones similares de nuestro medio. Lo ideal es transmitir el pensamiento colombiano para hacer honor al nombre de Sociedad de Urología Colombiana.

Han pasado muchos años sin que esta aspiración se cumpliera y hoy es una realidad gracias a la colaboración de Química Shering Colombiana y su director medico, el doctor Jaime Gaviria T., que no solo ha dado el respaldo económico, sino también sus ideas para concretar esta publicación. Aunque no está impresa a todo color, debido a los costos actuales, su contenido se presenta con gran entusiasmo a los urólogos colombianos con la esperanza de que en el futuro todos participen en ella y podamos mostrar a nivel nacional e internacional, la, hasta presente, inédita producción científica de la Urología Colombiana.

No deseo terminar esta nota editorial sin antes presentar un saludo a los colegas organizadores del congreso en la ciudad de Neiva, que estoy seguro será un éxito científico y social.
(C) 2021. Sociedad Colombiana de Urología. All rights reserved. This is an open access article published by Thieme under the terms of the Creative Commons Attribution-NonDerivative-NonCommercial-License, permitting copying and reproduction so long as the original work is given appropriate credit. Contents may not be used for commercial purposes, or adapted, remixed, transformed or built upon. (https://creativecommons.org/ licenses/by-nc-nd/4.0/)

Thieme Revinter Publicações Ltda., Rua do Matoso 170, Rio de Janeiro, RJ, CEP 20270-135, Brazil 

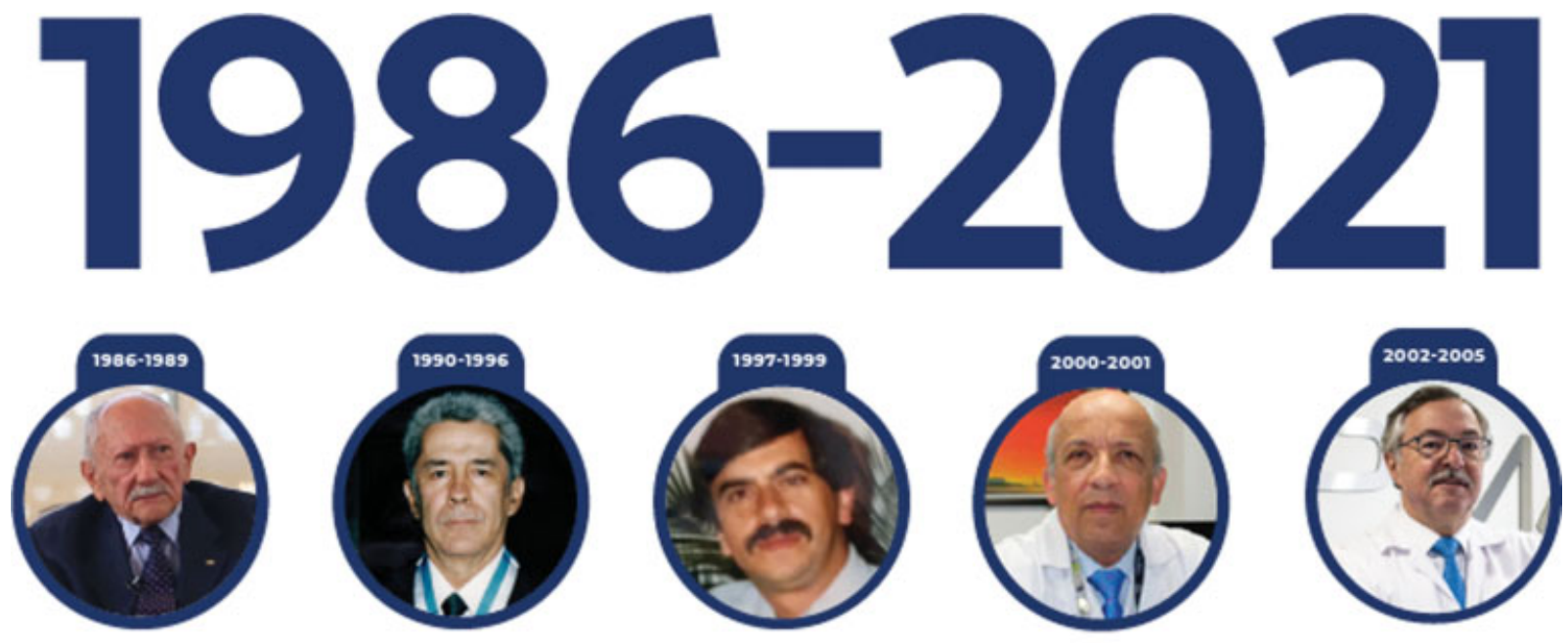

Dr. Armasde topez topez
(QtPD)

Dr. Jetés De Les Rios Ororio
(QtePO)

Dr. Jaime Diaz Berrocal

Dr. Jose Miguel Sillva Herrera

Dr. Pablo Comez Cusait

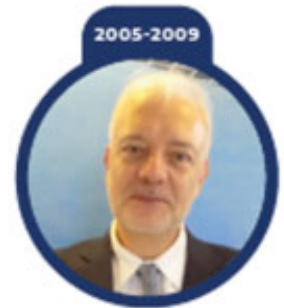

Dc. Juan Feraando Uribe

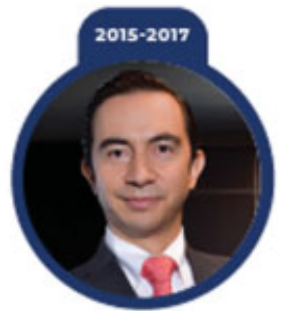

Dr. Huge Lopez Ramos

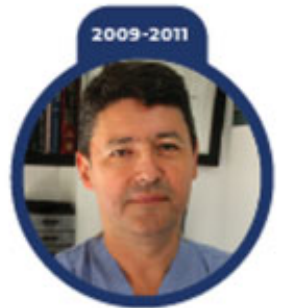

De. Mauricie Pata Salazar

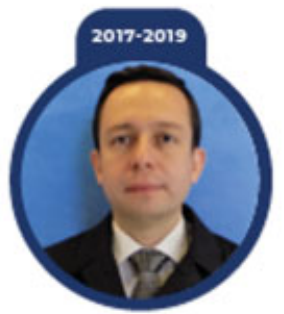

Dr. Jose Luis Poveda Matiz

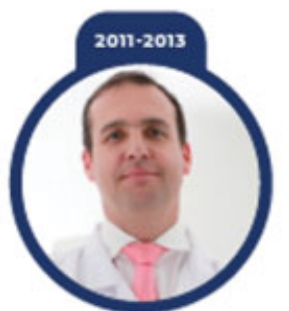

Dr. Carles Custave Trujlile Ordeftez

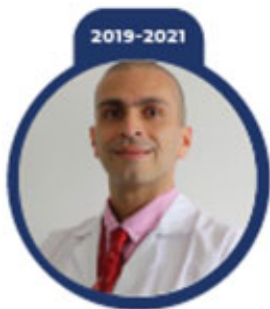

Dr. Herney Andres Carcia Perdomo

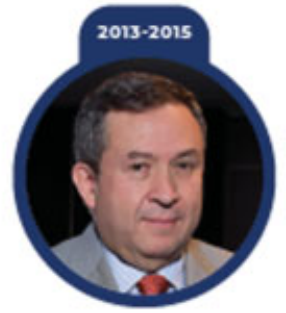

Dr. William Quiroga Matameros

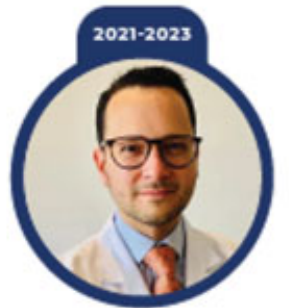

Dr. Aadres Felipe Cutlitrez Rojas
Agradecemos a los editores que han dedicado y aportado sus esfuerzos encaminados en sostener la publicación de RUC a lo largo del tiempo, ajustada a las exigencias y parámetros contemplados para las publicaciones científicas. 